\title{
Mutual Coupling Mechanisms Within Arrays of Nonlinear Antennas
}

\author{
Kun-Chou Lee, Senior Member, IEEE, and Tah-Hsiung Chu, Member, IEEE
}

\begin{abstract}
In this paper, mathematical analyses and physical interpretations for the mutual coupling mechanisms within a finite nonlinearly loaded antenna array are presented. These analyses are based on the method of nonlinear currents with the array mutual coupling effects treated by a power series expansion technique. Initially, the antenna input terminals in an antenna array are related by an equivalent multiport microwave circuit. Each port of this equivalent circuit network is then treated by the method of nonlinear currents. The antenna array mutual coupling effect is considered in this linear network and treated by a power series expansion technique. This study gives not only full analyses, but also physical insight into the mutual coupling mechanisms of nonlinearly loaded antenna arrays.
\end{abstract}

Index Terms-Loaded antenna, microwave circuit, mutual coupling,

\section{INTRODUCTION}

$\mathbf{M}$ UTUAL coupling effects of antenna arrays play a very important role in areas of electromagnetic compatibility (EMC) and electromagnetic interference (EMI). The existence of mutual coupling effects may affect the scattering and radiating characteristics of antenna arrays [1]-[4] and then degrade the performances of communication systems [5]-[7].

In some applications, nonlinear circuit devices are usually attached to the input terminal of an antenna for changing the scattering and radiating characteristics, i.e., the nonlinearly loaded antenna [8]. In [8], the problem of a single nonlinearly loaded antenna is transformed into an equivalent microwave circuit with the circuit parameters obtained by moment methods [9]. This transformation will make the analysis of such a problem very easy in the frequency domain. However, for array structures of nonlinearly loaded antennas, the mutual coupling effects are very difficult to analyze because of the nonlinear characteristics of each array element. In [10] and [11], the mutual coupling effects of nonlinearly loaded antenna arrays are approximated by the infinite periodic array method [12]-[14]. This treatment is suitable for analyzing a large periodic array by properly using the infinite periodic structure Green's function [12]-[14], in which the Poisson sum technique is applied to simplify the formulation, and the mutual coupling effects are automatically included

Manuscript received January 21, 2005; revised July 29, 2005. This work was supported by the National Science Council, Taiwan, R. O. C., under Grant NSC 94-2611-E-006-012.

K.-C. Lee is with the Department of Systems and Naval Mechatronic Engineering, National Cheng-Kung University, Tainan 701, Taiwan, R. O. C. (e-mail: kclee@mail.ncku.edu.tw).

T.-H. Chu is with the Department of Electrical Engineering and Graduate Institute of Communication Engineering, National Taiwan University, Taipei 106, Taiwan, R. O. C. (e-mail: thc@ew.ee.ntu.edu.tw).

Digital Object Identifier 10.1109/TEMC.2005.857892 in the resulting Green's function. Therefore, the analysis of an infinite antenna array will then become the analysis of a single antenna element with its free-space Green's function replaced by the modified Green's function of infinite structures. This approach reduces the computation effort considerably. However, it ignores the array edge effects and is limited to the periodic array structures only.

In this paper, mathematical analyses and physical interpretations for the mutual coupling mechanisms of a finite nonlinearly loaded antenna array are presented. To our knowledge, this paper is the first study to give full mathematical analyses and physical interpretations for mutual coupling effects within array structures of nonlinear antennas. According to [8], the analysis of a single nonlinearly loaded antenna can be transformed into an equivalent microwave circuit with the circuit element obtained by moment methods [9] and the steady-state scattering response is obtained efficiently in the frequency domain. The treatment of such equivalent microwave circuits can be extended to problems of array structures [11], [15], and [16]. The antenna input terminals within an antenna array are then related by an equivalent multiport microwave network and the array mutual coupling effects are included in the linear network. To clearly analyze the mutual coupling mechanisms, the analysis in [11], [15], and [16] for a finite nonlinearly loaded antenna array is reformulated based on the method of nonlinear currents [17] with the array mutual coupling effects treated by a power series expansion technique [18], [19]. The physical interpretations of the scattering mechanisms within array structures at different harmonic frequencies can then be given accordingly. In the analysis, the antenna input terminals in an antenna array are related by a multiport microwave network initially. Note that the antenna array mutual coupling effect is included in this linear network. To give physical insight into the mutual coupling mechanisms at different harmonic frequencies, each port of this equivalent circuit network is treated by the method of nonlinear currents [17]. This method is basically an alternative type of the Volterra series analysis [17] for microwave circuits. With the use of a power series technique [18], [19], the mutual coupling mechanisms within the nonlinearly loaded antenna array at each intermodulation frequency component are easily given accordingly.

Compared with our past studies in [11], [15], and [16], the method proposed in this paper facilitates the physical interpretation of the results. Furthermore, the method proposed here can give a specified frequency component of response alone without calculating the total responses. In other words, the proposed method can calculate, decompose, and then physically interpret the mutual coupling effects between nonlinear radiating 


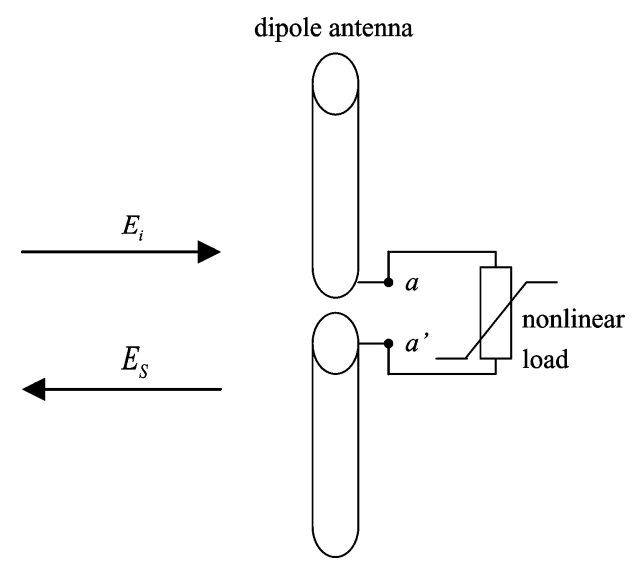

Fig. 1. Schematic diagram of a nonlinearly loaded dipole.

structures, whereas the methods given in [11], [15], and [16] can give only the total scattering response.

Nonlinear devices are often employed to protect electronic equipment against external power. Although nonlinearly loaded dipole antenna arrays are demonstrated in this paper, our proposed method has no limitation in types of antennas or nonlinear loads. In other words, the proposed method can be applied to any nonlinear radiating structure. This will make the proposed method useful in the area of electromagnetic compatibility.

In Section II, the analyses of a single nonlinearly loaded antenna are given by the method of nonlinear currents. These formulations are then extended to finite array structures in Section III. The physical interpretations for mutual coupling mechanisms within array structures are given in Section IV. Numerical examples are given in Section V. Finally, the conclusion is given in Section VI.

\section{SingLE ELEMENT}

To study the mutual coupling mechanisms of an array structure, we start from the behavior of a single antenna element. For simplicity, a single nonlinearly loaded dipole antenna illuminated by a plane wave $E_{i}$ is considered, as shown in Fig. 1 . According to [8], the equivalent microwave circuit of Fig. 1 is given as Fig. 2, where $i_{S}(t)$ is the current source induced by the incident wave, and $Y(\omega)$ is the antenna input admittance at frequency $\omega$. Note that the circuit parameters in Fig. 2 can be obtained from moment methods [9]. The symbol $\omega$ represents the frequency-domain variable throughout the paper. In other words, as we choose the fundamental frequency as $\omega_{0}$, then $\omega$ may be $\omega_{0}, 2 \omega_{0}, \ldots$, or $n \omega_{0}, \ldots$, etc.

The current-voltage $(i-v)$ characteristics of the nonlinear load can be described by

$$
i(t)=g_{1} v(t)+g_{2}[v(t)]^{2}+g_{3}[v(t)]^{3}+\Lambda \Lambda .
$$

According to the method of nonlinear currents given in [17], the voltage $v(t)$ in (1) consists of all orders of mixing frequency components, and is given as

$$
v(t)=v^{(1)}(t)+v^{(2)}(t)+v^{(3)}(t)+\Lambda \Lambda
$$

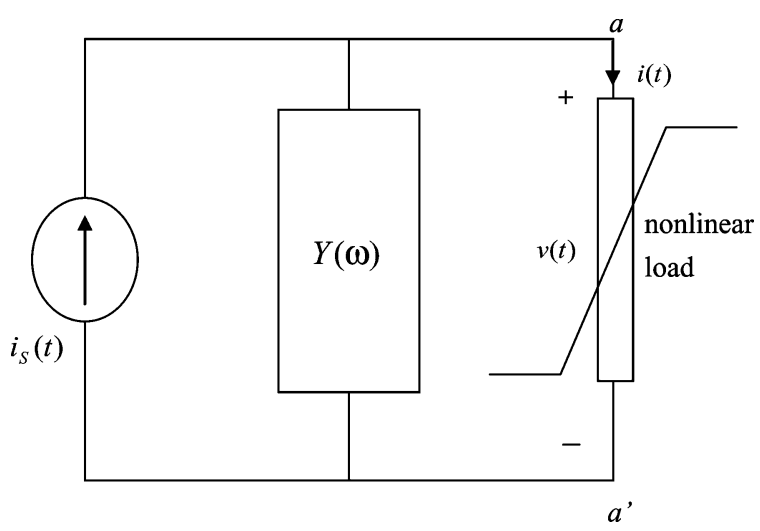

Fig. 2. Equivalent circuit of Fig. 1.

where $v^{(k)}(t)$ represents the sum of all $k$ th order mixing frequency components. The current $i(t)$ in (1) can be divided into a linear part $i_{\text {linear }}(t)$ and a nonlinear part $i_{\text {nonlinear }}(t)$, i.e.,

$$
i(t)=i_{\text {linear }}(t)+i_{\text {nonlinear }}(t)
$$

where

$$
i_{\text {linear }}(t)=g_{1} v(t)
$$

and

$$
i_{\text {nonlinear }}(t)=g_{2}[v(t)]^{2}+g_{3}[v(t)]^{3}+\Lambda .
$$

The linear part in (4) represents a linear resistor with resistance of $1 / g_{1}$. If the load nonlinearity in (5) is limited to the third degree, and (2) is only considered to the third-order mixing frequency components, substituting (2) into (5), we have

$$
\begin{aligned}
i_{\text {nonlinear }}(t)= & g_{2}\left[v^{(1)}(t)+v^{(2)}(t)+v^{(3)}(t)\right]^{2} \\
& +g_{3}\left[v^{(1)}(t)+v^{(2)}(t)+v^{(3)}(t)\right]^{3} \\
= & g_{2}\left\{\left[v^{(1)}(t)\right]^{2}+\left[v^{(2)}(t)\right]^{2}\right. \\
& +\left[v^{(3)}(t)\right]^{2}+2\left[v^{(1)}(t)\right]\left[v^{(2)}(t)\right] \\
& \left.+2\left[v^{(2)}(t)\right]\left[v^{(3)}(t)\right]+2\left[v^{(3)}(t)\right]\left[v^{(1)}(t)\right]\right\} \\
& +g_{3}\left\{\left[v^{(1)}(t)\right]^{3}+\left[v^{(2)}(t)\right]^{3}+\left[v^{(3)}(t)\right]^{3}\right. \\
& +3\left[v^{(1)}(t)\right]^{2}\left[v^{(2)}(t)\right]+3\left[v^{(1)}(t)\right]\left[v^{(2)}(t)\right]^{2} \\
& +3\left[v^{(2)}(t)\right]^{2}\left[v^{(3)}(t)\right]+3\left[v^{(2)}(t)\right]\left[v^{(3)}(t)\right]^{2} \\
& +3\left[v^{(3)}(t)\right]^{2}\left[v^{(1)}(t)\right]+3\left[v^{(3)}(t)\right]\left[v^{(1)}(t)\right]^{2} \\
& \left.+6\left[v^{(1)}(t)\right]\left[v^{(2)}(t)\right]\left[v^{(3)}(t)\right]\right\} .
\end{aligned}
$$

If (6) is only considered to the third-order mixing frequency components, we have

$$
\begin{aligned}
i_{\text {nonlinear }}(t) \cong & g_{2}\left\{\left[v^{(1)}(t)\right]^{2}+2\left[v^{(1)}(t)\right]\left[v^{(2)}(t)\right]\right\} \\
& +g_{3}\left\{\left[v^{(1)}(t)\right]^{3}\right\} \\
= & \left\{g_{2}\left[v^{(1)}(t)\right]^{2}\right\} \\
& +\left\{2 g_{2}\left[v^{(1)}(t)\right]\left[v^{(2)}(t)\right]+g_{3}\left[v^{(1)}(t)\right]^{3}\right\} .
\end{aligned}
$$

The nonlinear current source $i_{\text {nonlinear }}(t)$ in (7) can then be divided into the second-order mixing frequency component $i^{(2)}(t)$ 


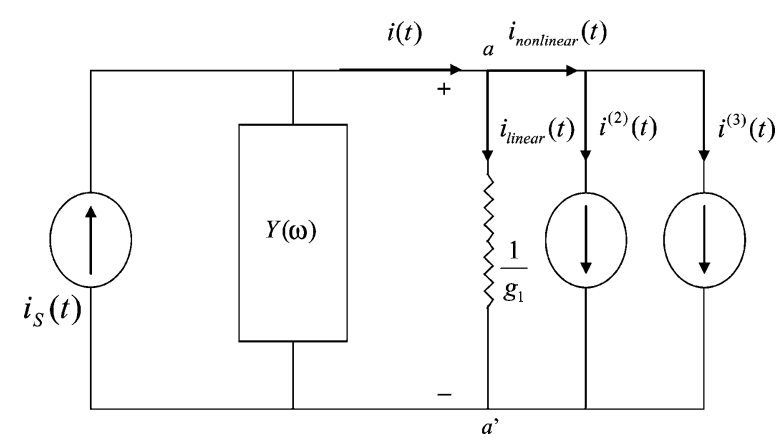

Fig. 3. Equivalent circuit of Fig. 2 based on the method of nonlinear currents.

and the third-order mixing frequency component $i^{(3)}(t)$, i.e.,

$$
i_{\text {nonlinear }}(t)=i^{(2)}(t)+i^{(3)}(t)
$$

where

$$
i^{(2)}(t)=g_{2}\left[v^{(1)}(t)\right]^{2}
$$

and

$$
i^{(3)}(t)=2 g_{2}\left[v^{(1)}(t)\right]\left[v^{(2)}(t)\right]+g_{3}\left[v^{(1)}(t)\right]^{3} .
$$

According to (1), (3), (4), and (8), the circuit in Fig. 2 can then be redrawn in Fig. 3.

The goal of our analyses is to obtain the terminal voltage of $a-a^{\prime}$, i.e., $v(t)$. By using the substitution theorem and setting all the current sources except $i_{S}(t)$ to be zero initially, i.e., $i^{(2)}(t)=$ 0 and $i^{(3)}(t)=0$, one can obtain the voltage component from the contribution of $i_{S}(t)$ only. This voltage component is regarded as $v^{(1)}(t)$ in (9) and (10). The total iteration sequence is given as

$$
\begin{array}{r|l}
v^{(1)} & \rightarrow i^{(2)} \rightarrow v^{(2)} \rightarrow i^{(3)} \rightarrow v^{(3)} \rightarrow \cdots \\
\text { linearpart } \Rightarrow & \Leftarrow \text { nonlinearpart (method of nonlinear currents) }
\end{array}
$$

For example, assume a multitone excitation exists as

$$
i_{S}(t)=\sum_{\substack{q=-Q \\ q \neq 0}}^{Q} I_{q}^{S} e^{j \omega_{q} t}
$$

By setting all the current sources except $i_{S}(t)$ to be zero initially, one can obtain

$$
v^{(1)}(t)=\sum_{\substack{q=-Q \\ q \neq 0}}^{Q} V_{q}^{(1)} e^{j \omega_{q} t}
$$

where $V_{q}^{(1)}=\left(I_{q}^{S}\right) /\left(Y\left(\omega_{q}\right)+g_{1}\right)$. From (9), the current source $i^{(2)}(t)$ becomes

$$
i^{(2)}(t)=g_{2} \sum_{\substack{q_{1}=-Q \\ q_{1} \neq 0}}^{Q} \sum_{\substack{q_{2}=-Q \\ q_{2} \neq 0}}^{Q} V_{q_{1}}^{(1)} V_{q_{2}}^{(1)} e^{j\left(\omega_{q_{1}}+\omega_{q_{2}}\right) t} .
$$

Similarly by setting all current sources except $i^{(2)}(t)$ to be zero, one can obtain

$$
v^{(2)}(t)=-\sum_{\substack{q_{1}=-Q \\ q_{1} \neq 0}}^{Q} \sum_{\substack{q_{2}=-Q \\ q_{2} \neq 0}}^{Q} \frac{g_{2} V_{q_{1}}^{(1)} V_{q_{2}}^{(1)}}{Y\left(\omega_{q_{1}}+\omega_{q_{2}}\right)+g_{1}} e^{j\left(\omega_{q_{1}}+\omega_{q_{2}}\right) t} .
$$

From (10)

$$
\begin{aligned}
i^{(3)}(t)= & 2 g_{2} \sum_{\substack{q_{1}=-Q \\
q_{1} \neq 0}}^{Q} \sum_{\substack{q_{2}=-Q \\
q_{2} \neq 0}}^{Q} \sum_{\substack{q_{3}=-Q \\
q_{3} \neq 0}}^{Q} \frac{-g_{2} V_{q_{1}}^{(1)} V_{q_{2}}^{(1)} V_{q_{3}}^{(1)}}{Y\left(\omega_{q_{2}}+\omega_{q_{3}}\right)+g_{1}} \\
& \times e^{j\left(\omega_{q_{1}}+\omega_{q_{2}}+\omega_{q_{3}}\right) t} \\
& +g_{3} \sum_{\substack{q_{1}=-Q \\
q_{1} \neq 0}}^{Q} \sum_{\substack{q_{2}=-Q \\
q_{2} \neq 0}}^{Q} \sum_{\substack{q_{3}=-Q \\
q_{3} \neq 0}}^{Q} V_{q_{1}}^{(1)} V_{q_{2}}^{(1)} V_{q_{3}}^{(1)} \\
& \times e^{j\left(\omega_{q_{1}}+\omega_{q_{2}}+\omega_{q_{3}}\right) t}
\end{aligned}
$$

and the third-order voltage caused by $i^{(3)}(t)$ is then given as

$$
\begin{aligned}
v^{(3)}(t)= & 2 g_{2} \sum_{\substack{q_{1}=-Q \\
q_{1} \neq 0}}^{Q} \sum_{\substack{q_{2}=-Q \\
q_{2} \neq 0}}^{Q} \sum_{\substack{q_{3}=-Q \\
q_{3} \neq 0}}^{Q} \frac{g_{2} V_{q_{1}}^{(1)} V_{q_{2}}^{(1)} V_{q_{3}}^{(1)}}{Y\left(\omega_{q_{2}}+\omega_{q_{3}}\right)+g_{1}} \\
& \times \frac{e^{j\left(\omega_{q_{1}}+\omega_{q_{2}}+\omega_{q_{3}}\right) t}}{Y\left(\omega_{q_{1}}+\omega_{q_{2}}+\omega_{q_{3}}\right)+g_{1}} \\
& -g_{3} \sum_{\substack{q_{1}=-Q \\
q_{1} \neq 0}}^{Q} \sum_{\substack{q_{2}=-Q \\
q_{2} \neq 0}}^{Q} \sum_{\substack{q_{3}=-Q \\
q_{3} \neq 0}}^{Q} \frac{V_{q_{1}}^{(1)} V_{q_{2}}^{(1)} V_{q_{3}}^{(1)}}{Y\left(\omega_{q_{1}}+\omega_{q_{2}}+\omega_{q_{3}}\right)+g_{1}} \\
& \times e^{j\left(\omega_{q_{1}}+\omega_{q_{2}}+\omega_{q_{3}}\right) t} .
\end{aligned}
$$

The total voltage response $v(t)$ can be found from (2), (13), (15), and (17). The scattering or radiating characteristics of the antenna can then be determined accordingly.

\section{ARRAY StRUCTURES AND MUTUAL COUPLING MECHANISMS}

Consider an $N$-element nonlinearly loaded dipole antenna array illuminated by a plane wave $E_{i}$, as shown in Fig. 4. According to [11], [15], and [16], the relation between these $N$-element feeding terminals can be viewed as an $N$-port microwave network denoted by the array input admittance matrix $\bar{Y}(\omega)$. Similar to the treatment of single antenna element in Section II, the equivalent circuit of Fig. 4 is shown in Fig. 5 with the network parameters obtained by moment methods [9]. Note that the antenna array mutual coupling effect is included in this linear network. In Fig. $5, I_{P}^{S}(\omega)$ is phasor representation of the equivalent current source at port $P$ due to the incident wave. Similarly, $I_{P}^{(k)}(\omega)$ and $V_{P}^{(k)}(\omega)$ are phasor representations of the $k$ th order mixing the frequency component of current and voltage at port $P$, respectively. Similar to the iteration procedure of the single antenna element in Section II, one can first set all the current sources except $I_{P}^{S}(\omega), P=1, \ldots, N$, to be zero. 


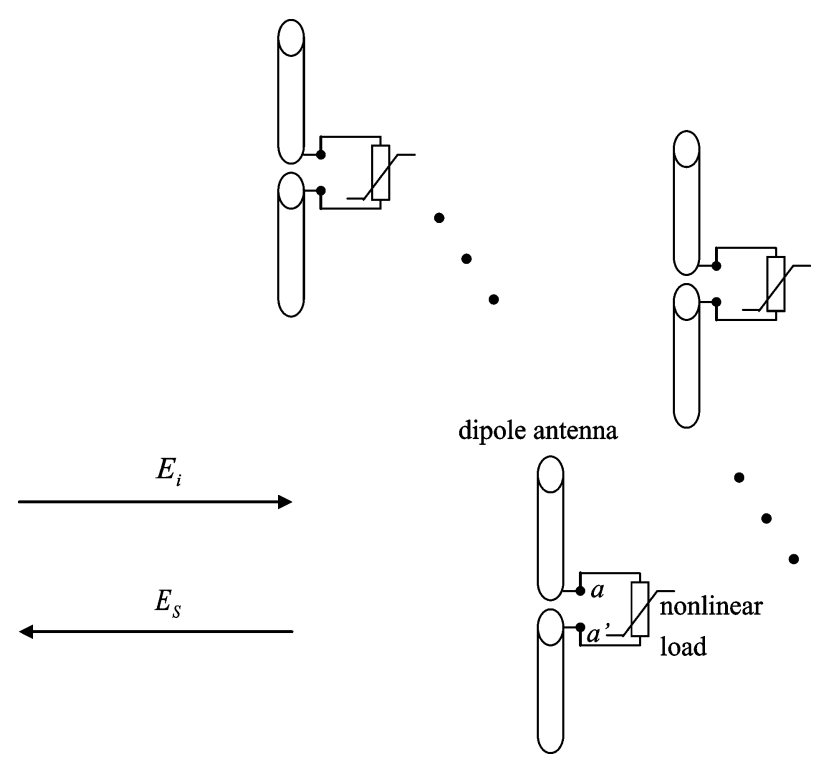

Fig. 4. Schematic diagram of a nonlinearly loaded dipole antenna array.

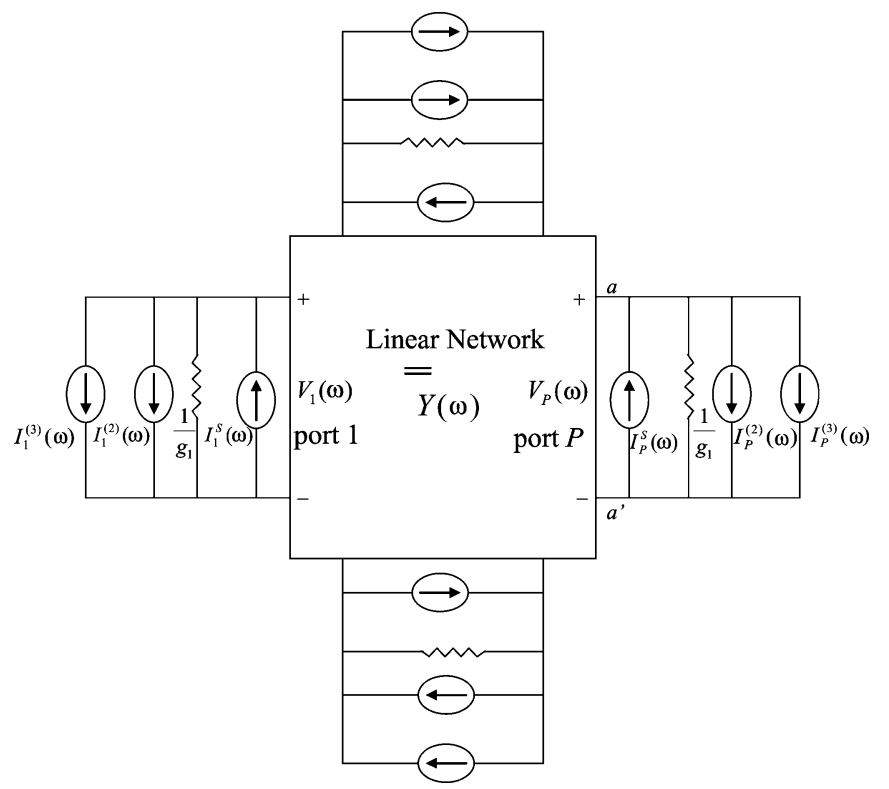

Fig. 5. Equivalent circuit representation of Fig. 4, with each current source representing the same order of mixing products, and $P=1,2, \ldots, N$.

From $\mathrm{KCl}$, one can write

$$
\bar{I}^{S}(\omega)=\left[\overline{\bar{Y}}(\omega)+g_{1} \overline{\bar{U}}\right] \bar{V}^{(1)}(\omega)
$$

then

$$
\bar{V}^{(1)}(\omega)=\left[\overline{\bar{Y}}(\omega)+g_{1} \overline{\bar{U}}\right]^{-1} \bar{I}^{S}(\omega)
$$

where $\overline{\bar{U}}$ is an $N \times N$ unit matrix

$$
\bar{I}^{S}(\omega)=\left[\begin{array}{lllll}
I_{1}^{S}(\omega) & \Lambda & I_{P}^{S}(\omega) & \Lambda & I_{N}^{S}(\omega)
\end{array}\right]^{T}
$$

and

$$
\bar{V}^{(1)}(\omega)=\left[\begin{array}{lllll}
V_{1}^{(1)}(\omega) & \Lambda & V_{P}^{(1)}(\omega) & \Lambda & V_{N}^{(1)}(\omega)
\end{array}\right]^{T} .
$$

Obviously, (20) and (21) are phasor representations of excitation current sources due to the incident field and the first-order mixing frequency voltage at each port, respectively. The $(i, j)$ th element of $Y(\omega), Y_{i, j}(\omega)$, is the mutual admittance between the $i$ th and the $j$ th antenna elements. The first-order mixing voltage at port $P v_{P}^{(1)}(t)$ can then determined from (21) by using inverse Fourier transformation.

Similarly, for order $k \geq 2$ one can set all sources except $I_{P}^{(k)}(\omega), P=1, \ldots, N$, to be zero, and obtain

$$
-\bar{I}^{(k)}(\omega)=\left[\overline{\bar{Y}}(\omega)+g_{1} \overline{\bar{U}}\right] \bar{V}^{(k)}(\omega)
$$

then

$$
\bar{V}^{(k)}(\omega)=-\left[\overline{\bar{Y}}(\omega)+g_{1} \overline{\bar{U}}\right]^{-1} \bar{I}^{(k)}(\omega)
$$

Similar to (20)-(21), $\bar{I}^{(k)}(\omega)$ and $\bar{V}^{(k)}(\omega)$ are phasor representations of the $k$ th order mixing frequency current and voltage at each port, respectively. Therefore, the $k$ th order mixing components $i_{P}^{(k)}(t)$ and $v_{P}^{(k)}(t)$ at port $P$ can be determined accordingly.

Similar to the treatment in [19], for identical array elements, the array input impedance matrix $\overline{\bar{Z}}(\omega)$ can be written as $Z_{\text {iso }}(\omega)[\overline{\bar{U}}+\overline{\bar{M}}(\omega)]$, where $Z_{\text {iso }}(\omega)$ is the input impedance of an isolated antenna element, and $\overline{\bar{M}}(\omega)$ is a dimensionless matrix to account for the mutual coupling effects with its elements given as

$$
M_{i, j}(\omega)= \begin{cases}0, & \text { for } i=j \\ Z_{i, j}(\omega) / Z_{\text {iso }}(\omega), & \text { otherwise. }\end{cases}
$$

The $Z_{i, j}(\omega)$ in (24) is the $(i, j)$ th element of $\overline{\bar{Z}}(\omega)$ and is the mutual impedance between the $i$ th and the $j$ th antenna elements. The $\overline{\bar{Y}}(\omega)$ in (18), (19), (22), and (23) can then be expanded as

$$
\begin{aligned}
\overline{\bar{Y}}(\omega) & =[\overline{\bar{Z}}(\omega)]^{-1} \\
& =\frac{1}{Z_{\text {iso }}(\omega)}\left\{\overline{\bar{U}}-\overline{\bar{M}}(\omega)+[\overline{\bar{M}}(\omega)]^{2}-[\overline{\bar{M}}(\omega)]^{3}+\Lambda\right\} .
\end{aligned}
$$

The matrix inversion in (19) and (23) can be rewritten as

$$
\begin{aligned}
& {\left[\overline{\bar{Y}}(\omega)+g_{1} \overline{\bar{U}}\right]^{-1}=r Z_{\text {iso }}(\omega)} \\
& \quad\left\{\overline{\bar{U}}+r \sum_{n=1}^{\infty}(-1)^{n-1}[\overline{\bar{M}}(\omega)]^{n}(1-r)^{n-1}\right\}
\end{aligned}
$$

where $r=(1) /\left(g_{1} Z_{\text {iso }}(\omega)+1\right)$. From (19), (23)-(26), the phasor representation of the $k$ th-order mixing frequency voltage for the dipole antenna $P$ can be expressed as

$$
\begin{aligned}
V_{P}^{(k)}(\omega) & =\left\{-r Z_{\text {iso }}(\omega) I_{P}^{(k)}(\omega)\right\} \\
+ & \left\{-r Z_{\text {iso }}(\omega) \sum_{\substack{i \\
i \neq P}} M_{P, i}(\omega) r I_{i, k}(\omega)\right\}
\end{aligned}
$$




$$
\begin{gathered}
+\left\{r Z_{\text {iso }}(\omega)(1-r) \sum_{\substack{j \\
j \neq P}} \sum_{\substack{i \\
i \neq j}} M_{P, j}\right. \\
\left.\times(\omega) M_{j, i}(\omega) r . I_{i}^{(k)}(\omega)\right\} \\
+\left\{\begin{array}{c}
-r Z_{\text {iso }}(\omega)(1-r)^{2} \sum_{\substack{l \\
l \neq P}} \sum_{\substack{j \\
j \neq l}} \sum_{\substack{i \\
i \neq j}} M_{P, l}(\omega) M_{l, j} \\
\left.\times(\omega) M_{j, i}(\omega) r I_{i}^{(k)}(\omega)\right\}+\cdots
\end{array}\right.
\end{gathered}
$$

The total voltage response $v_{P}(t)$ at each port, i.e., antenna terminal voltage, can be found from (2), (19), and (23)-(27). The scattering or radiating characteristics of the antenna array can then be determined accordingly.

\section{PhysicAl InTERPRETATIONS OF MUtUAL COUPLING MECHANISMS}

Based on the formulations given above, the $k$ th order mixing frequency component of the induced voltage $v_{P}^{(k)}(t)$ across the terminal of port $P$ can be found from (19), (23)-(27) and the inverse Fourier transformation. These voltage spectrum components can produce the reradiating fields at each intermodulation harmonic frequency. With the use of the method of nonlinear currents, the current source components are obtained from the voltage components of lower order mixing frequencies. Voltage components of the same order mixing frequency are then determined from these currents, and the current components of the next higher order mixing frequency can be found successively. These properties are shown in (11). It should be emphasized that each order of frequency component of the scattering response can be determined directly instead of calculating the total scattering response.

The physical meanings of $[\overline{\bar{M}}(\omega)]^{n}$ and the sign in (25) are given in detail in [19]. As shown in Fig. 6, the term $M_{i, j}(\omega)$ represents the direct coupling on the $i$ th dipole from the $j$ th dipole. The term $M_{i, j}(\omega) M_{j, k}(\omega)$ represents the "two-trip" coupling from the $k$ th dipole to the $j$ th dipole then to the $i$ th dipole. Similarly, the term of (27a) is the induced voltage of the $k$ th order mixing frequency at dipole $P$ without any mutual coupling. The term $r Z_{\text {iso }}(\omega)$ in (26) and (27) is equal to the shunt impedance of $(1) /\left(g_{1}\right)$ and $Z_{\text {iso }}(\omega)$, which is the result of the isolated element with linear approximation of the nonlinear lumped load, whereas the term of (27b) represents the first-order mutual coupling, which means the induced voltage due to the direct coupling from the other $N-1$ antennas. Similarly, the term of (27c) stands for the contribution of "two-trip" mutual coupling, and the term of (27d) is the "triple-trip" coupling effect. Since the current source components are obtained from voltage components of lower order mixing frequencies in the formulations, the array mutual coupling effects are affected by those of lower

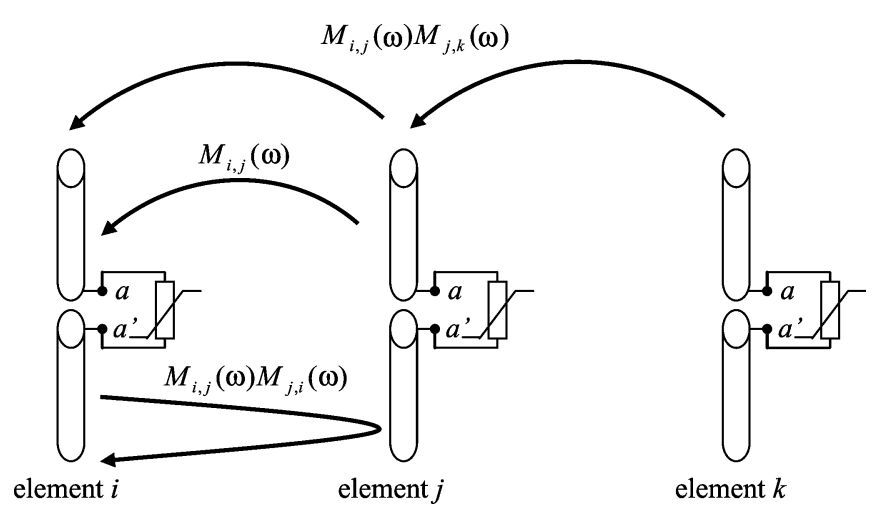

Fig. 6. Interpretations of mutual coupling mechanisms within a nonlinearly loaded antenna array.

order mixing frequencies. These interpretations of mutual coupling mechanisms are consistent with those given in [20].

\section{NUMERICAL EXAMPLES}

In this section, numerical examples are given to illustrate the scattering mechanisms given above. For simplicity, dipole antennas with each input terminal loaded with a lumped load are considered. Each dipole is $1 \mathrm{~m}$ long and has length-to-diameter ratio of 74.2. The incident plane wave is monochrome and has strength $E_{i}=1.0 \mathrm{~V} / \mathrm{m}$. The $i-v$ characteristics of the lumped load at the input terminal of each dipole is nonlinear and given as

$$
i(t)=\frac{1}{75} v(t)+4 v^{3}(t)
$$

The nonlinearity above can be implemented by two parallel diodes with opposite directions. Note that the proposed method has no limitation on the types of nonlinear loads. In this section, we choose the coefficients in (1) as $g_{1}=(1 / 75), g_{3}=4$ and the other terms to be zero. These parameters are the same as those given in [8], [10], [11], and [16] so that the numerical results of different papers can be compared under the same parameters. In our treatment of mutual coupling within a nonlinearly loaded antenna array, the mutual impedances between any two antenna elements within the array are required. We utilize moment methods to obtain these required mutual impedances. The mutual impedances calculated by our moment method programs have been compared with the examples in [21], [22] and all were found to be consistent.

In the first numerical example, a single nonlinearly loaded antenna illuminated by monochrome plane waves of different frequencies is considered. Following the analysis given in Section II, the backscattered radar cross section (RCS) of fundamental frequency is shown in Fig. 7. Note that the results by the method of nonlinear currents described in Section II are consistent with [8, Fig. 4] that treats the same problem by the harmonic balance technique [17]. From this example, we are convinced that the method of nonlinear currents gives accurate results in problems of nonlinearly loaded antennas. It should be emphasized that the purpose of this example is to verify the correctness 


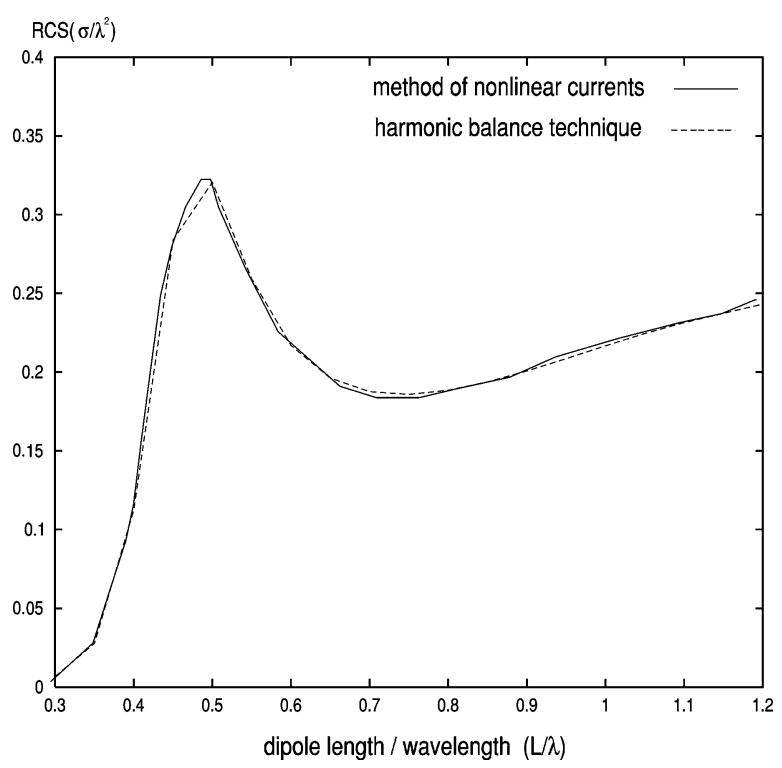

Fig. 7. Comparison for the results of RCS of a single nonlinearly loaded antenna by using harmonic balance technique and methods of nonlinear currents.

for the use of methods of nonlinear currents described above in this type of problem.

In the second numerical example, a nonlinearly loaded antenna array consisting of two parallel dipoles normally illuminated by a plane wave of frequency $140 \mathrm{MHz}$ is considered. Each array element is the same as that given in the first numerical example. Because of the symmetry of this two-dipole arrangement, the resulting voltage at each antenna terminal is the same. The exact voltage $V_{\text {exact }}$ at each antenna terminal is calculated by using the left side of (26). Obviously, $V_{\text {exact }}$ contains all orders of mutual coupling mechanisms in (25). The approximate voltage $V_{\text {model }}$ at each antenna terminal is calculated by evaluating the mutual coupling mechanisms of (26) or (25) without mutual coupling [i.e., the right side of (26) contains $\overline{\bar{U}}$ only, or (27a)], to the first-order mutual coupling (i.e., the right side of (26) contains $\overline{\bar{U}}$ and $n=1$ terms, or $(27 a)+(27 b))$, to the second-order mutual coupling [i.e., the right side of (26) contains $\overline{\bar{U}}, n=1$ and $n=2$ terms, or (27a) + $(27 b)+(27 c)]$, and to the third-order mutual coupling [i.e., the right side of (26) contains $\overline{\bar{U}}, n=1, n=2$, and $n=3$ terms, or $(27 a)+(27 b)+(27 c)+(27 d)]$, respectively. The relative percentage error of antenna terminal voltage is defined as

$$
\% \text { Error }=\frac{\left|V_{\text {model }}\right|-\left|V_{\text {exact }}\right|}{\left|V_{\text {exact }}\right|} \times 100 \% \text {. }
$$

Fig. 8 shows the relative percentage error of antenna terminal voltage under the different element spacing of side-by-side array geometry. It shows that $V_{\text {model }}$ becomes closer to $V_{\text {exact }}$ as the array element spacing increases and good accuracy is obtained in this problem by considering $V_{\text {model }}$ to the third-order mutual coupling, i.e., higher order coupling terms have little contribution. It should be emphasized that the purpose of this example is to demonstrate the mutual coupling mechanisms within a finite nonlinearly loaded antenna array and the contribution from each order of mutual coupling.

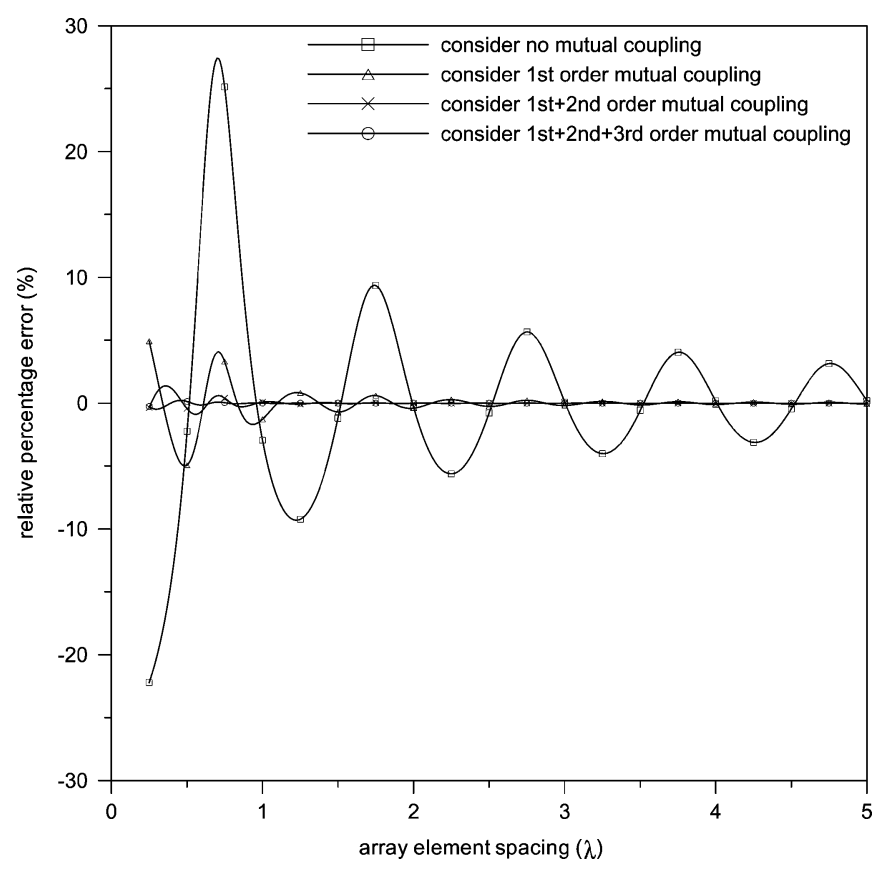

Fig. 8. Relative percentage error of antenna terminal voltage under different element spacing of side-by-side array geometry.

It is well known that many practical arrays are collinear arrays. In the third example, the side-by-side array geometry of the second example is replaced by the collinear array geometry. All the parameters are the same as those given in the second example. Fig. 9 shows the relative percentage error of antenna terminal voltage under different element spacing of collinear array geometry. In general, the results of mutual coupling in Fig. 9 are weaker than those of Fig. 8. This is because a $\hat{z}$-direction dipole produces $\hat{\theta}$-direction electric fields. These $\hat{\theta}$-direction electric fields will then induce larger currents on other dipoles of side-by-side direction than of collinear direction.

To show the dependence of our results on the chosen nonlinearity of (28), the magnitude of voltage at each input terminal of two side-by-side nonlinearly loaded dipoles for different array element spacing at different harmonic frequencies of $1 \omega, 2 \omega$, and $3 \omega$ are calculated using the formulations in Sections II and III. The mutual coupling is considered to the second-order term. Note that the results of Fig. 10 are consistent with [16, Fig. 4] that treats the same problem by the neural network and harmonic balance techniques. Fig. 10 shows some nonlinear phenomena resulted from the nonlinearity of (28). Due to the linear $(v)$ term in (28), the voltage component at fundamental frequency $\omega$ is much greater than those of the higher order mixing frequencies. In addition, due to the effect of the cubic $\left(v^{3}\right)$ term in (28), the voltage component at mixing frequency $3 \omega$ is slightly greater than those at mixing frequency $2 \omega$.

The numerical simulation above is executed on a personal computer with CPU of Intel Pentium $2.8 \mathrm{GHz}$. Fig. 11 shows the computation time for the above two-element array structures spaced by a given distance by considering different order terms of mutual coupling. Although the computation time increases with the considered order terms of mutual coupling, 


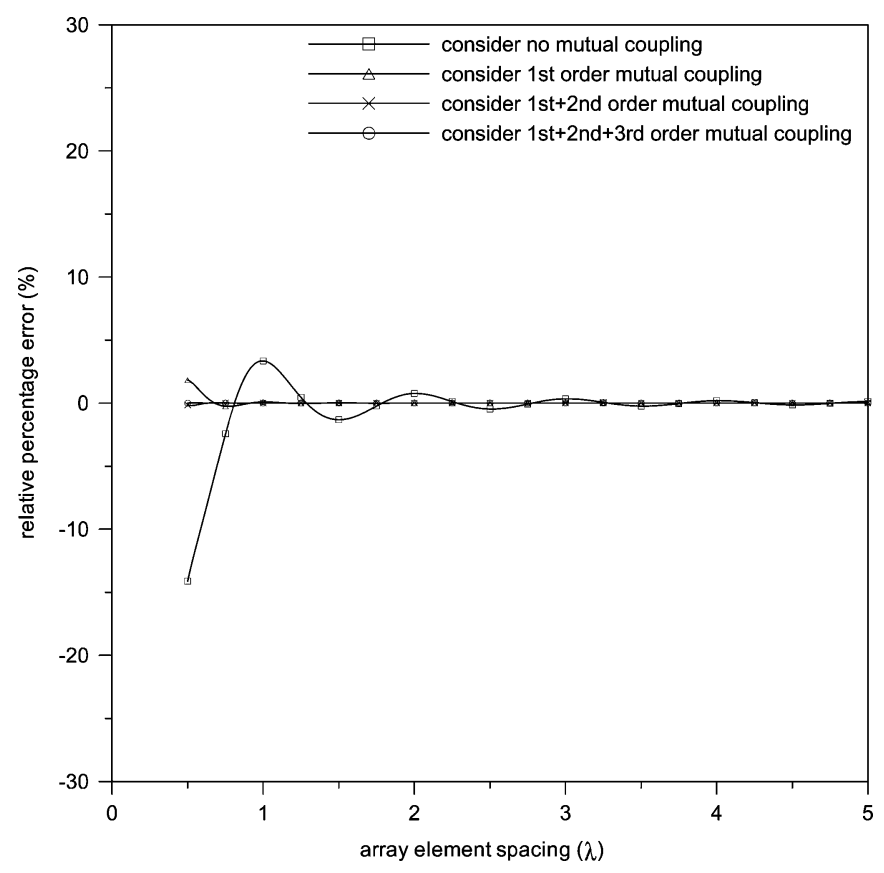

Fig. 9. Relative percentage error of antenna terminal voltage under different element spacing of collinear array geometry.

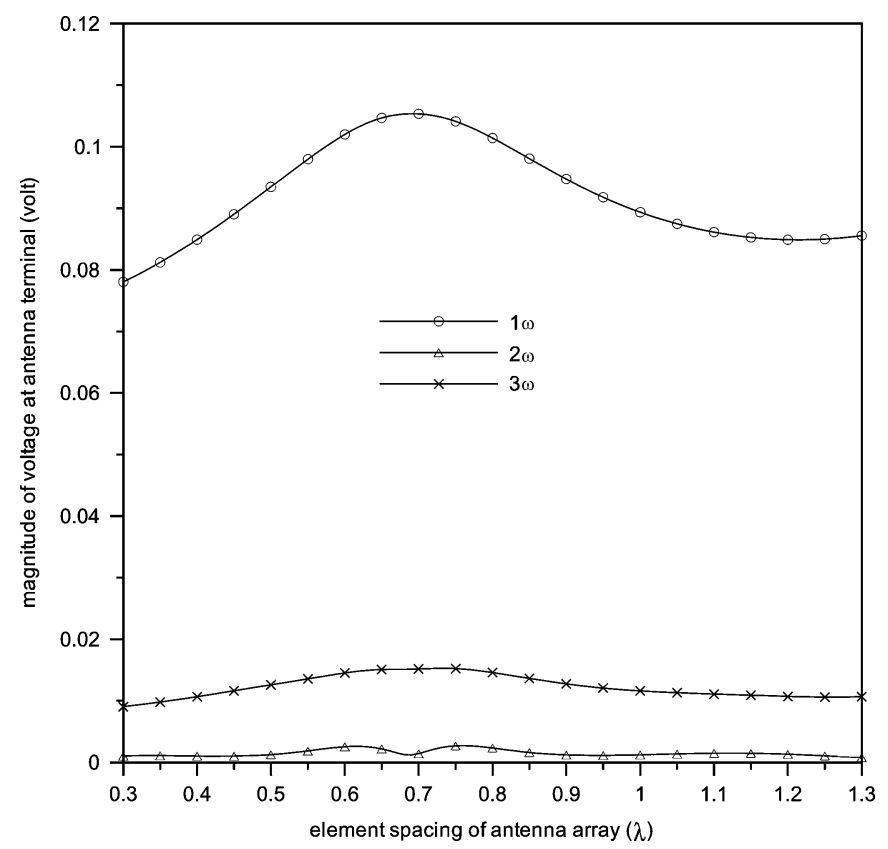

Fig. 10. Magnitude of voltage at each input terminal of two side-by-side nonlinearly loaded dipoles for different array element spacing at different harmonic frequencies of $1 \omega, 2 \omega$, and $3 \omega$.

from the results of Figs. 8-10, it is sufficiently accurate to model the mutual coupling to the second-order terms.

\section{CONCLUSION}

In this paper, the mutual coupling mechanisms of a finite nonlinearly loaded antenna array are given based on the method of nonlinear currents with the array mutual coupling effects treated by a power series expansion technique. Note that the results

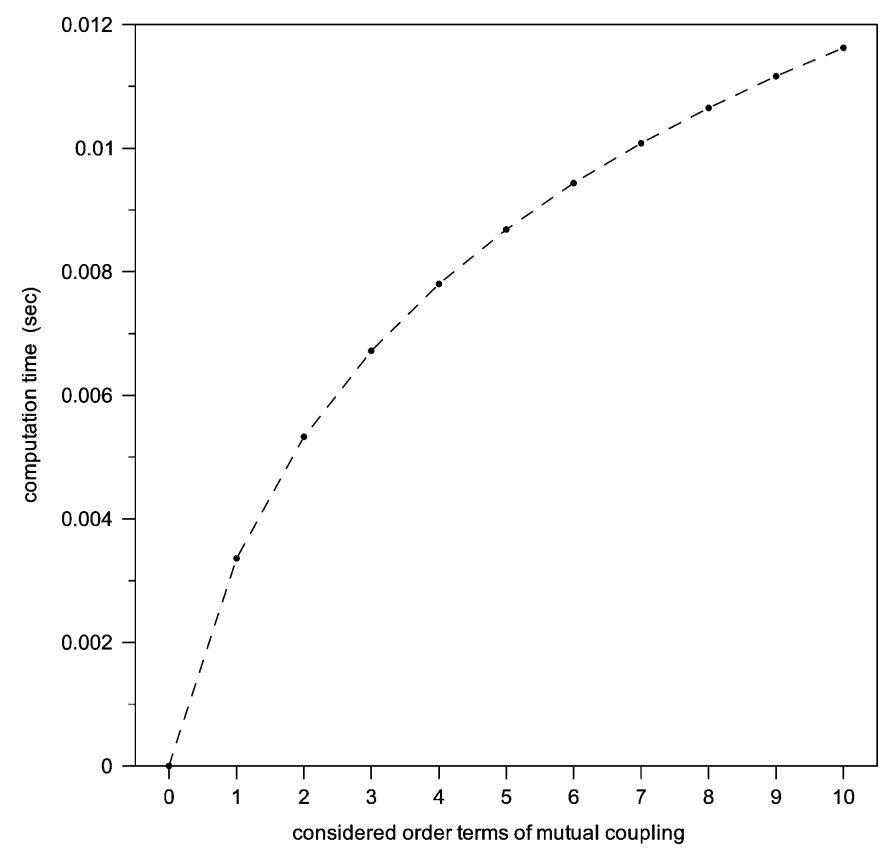

Fig. 11. Computation time for the above two-element array structures spaced by a given distance by considering different order terms of mutual coupling.

of mathematical formulations for total voltage response given above can be proved to be equivalent to those by the Volterra series approach in [17]. However, physically, some differences exist between these two approaches. The approach in this paper can be easily combined with the circuit model for mutual coupling in [19] to interpret the mutual coupling mechanisms within a finite nonlinearly loaded antenna array. By using the method of nonlinear currents, each order of current is determined by voltages with lower order mixing frequencies. With the use of the method of nonlinear currents, the mutual coupling mechanisms within a finite nonlinearly loaded array can be interpreted through different mixing orders of equivalent circuits, as given in Section IV. Because the array mutual coupling effects are treated by a power series technique, they can be divided into various orders of contributions. In summary, one can analyze and give physical insight into the mutual coupling mechanisms of a finite nonlinearly loaded antenna array at each order of mixing frequencies through the approaches proposed in this paper. This study will be useful in the areas of nonlinear antennas and electromagnetic compatibility.

\section{ACKNOWLEDGMENT}

The authors would like to express their sincere gratitude to the National Center for High-Performance Computing, Taiwan, R. O. C., for the support of computing software.

\section{REFERENCES}

[1] B. J. Strait and A. T. Adams, "Analysis and design of wire antennas with applications to EMC," IEEE Trans. Electromagn. Compat., vol. EMC-12, no. 2, pp. 45-54, May 1970.

[2] J. Perini and K. Hirasawa, "Antenna pattern distortion and mutual coupling in antenna farms," in IEEE Electromagn. Compat. Symp. Rec., Jun. 1973, pp. 201-208. 
[3] K. R. Siarkiewicz and A. T. Adams, "Near and far field thin wire coupling-theory and experiment," IEEE Trans. Electromagn. Compat., vol. EMC-19, no. 4, pp. 394-401, Nov. 1977.

[4] R. Luebbers and K. Kunz, "Finite difference time domain calculations of antenna mutual coupling," IEEE Trans. Electromagn. Compat., vol. 34 no. 3, pp. 357-359, Aug. 1992.

[5] B. Keiser, Principles of Electromagnetic Compatibility. Norwood, MA: Artech House, 1987.

[6] K. Hirasawa, "Bounds of uncertain interference between closely located antennas," IEEE Trans. Electromagn. Compat., vol. EMC-26, no. 3, pp. 129-133, Aug. 1984.

[7] G. Y. Delisle and J. A. Cummins, "Mutual coupling in the signal-to-noise ratio optimization of antenna arrays," IEEE Trans. Electromagn. Compat., vol. EMC-15, no. 2, pp. 38-44, May 1973.

[8] C. C. Huang and T. H. Chu, "Analysis of wire scatterers with nonlinear or time-harmonic loads in the frequency domain," IEEE Trans. Antennas Propagat., vol. 41, no. 1, pp. 25-30, Jan. 1993.

[9] R. F. Harrington, Field Computation by Moment Methods. New York: Macmillan, 1968.

[10] K. C. Lee, "Two efficient algorithms for the analyses of a nonlinearly loaded antenna and antenna array in the frequency domain," IEEE Trans. Electromagn. Compat., vol. 42, no. 4, pp. 339-346, Nov. 2000.

[11] _ "Genetic algorithms based analyses of nonlinearly loaded antenna arrays including mutual coupling effects," IEEE Trans. Antennas Propag., vol. 51, no. 4, pp. 776-781, Apr. 2003.

[12] A. A. Oliner and R. G. Malech, "Mutual coupling in infinite scanning arrays," in Microwave Scanning Antennas, R. C. Hansen, Ed. New York: Academic, 1966, ch. 3, pp. 195-335.

[13] L. Stark, "Radiation impedance of a dipole in an infinite planar phased array," Radio Sci., vol. 1, pp. 361-377, Mar. 1966.

[14] D. M. Pozar and D. H. Schaubert, "Scan blindness in infinite phased arrays of printed dipoles," IEEE Trans. Antenna Propag., vol. AP-32, no. 6, pp. 602-610, Jun. 1984.

[15] K. C. Lee and T. H. Chu, "Analysis of injection-locked antenna array including mutual coupling effects," IEEE Trans. Antennas Propag., vol. 52, no. 11 , pp. 2885-2890, Nov. 2004.

[16] _ - "Application of neural networks to analyses of nonlinearly loaded antenna arrays including mutual coupling effects," IEEE Trans. Antennas Propag., vol. 53, no. 3, pp. 1126-1132, Mar. 2005.

[17] S. A. Mass. Nonlinear Microwave Circuits. Norwood, MA: Artech House, 1988, ch. 4.

[18] D. Westreich, "Evaluating the matrix polynomial $I+A+A^{2}+$ $+A^{N-1}, "$ IEEE Trans. Circuits Syst., vol. 36, no. 1, pp. 162-164, Jan. 1989.
[19] K. C. Lee and T. H. Chu, "A circuit model for mutual coupling analysis of a finite antenna array," IEEE Trans. Electromagn. Compat., vol. 38, no. 3, pp. 483-489, Aug. 1996.

[20] V. Twersky, "Multiple scattering of radiation by arbitrary configuration of parallel cylinders," J. Acoust. Soc. Amer., vol. 24, no. 1, pp. 42-46, Jan. 1952.

[21] 7.15R. S. Elliot, Antenna Theory and Design, New York: Wiley, 2003.

[22] J. D. Krous and R. J. Marhefka. Antennas-For all Application. New York: McGraw-Hill, 2003, ch. 13.

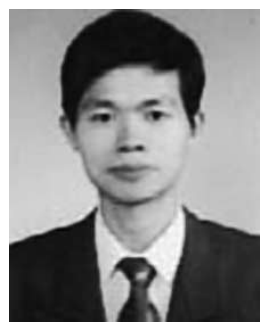

Kun-Chou Lee (M'00-SM'05) was born in Chiayi, Taiwan, R. O. C., in 1966. He received the B.S., M.S., and Ph.D. degrees from the National Taiwan University, Taipei, Taiwan, in 1989, 1991, and 1995, respectively, all in electrical engineering.

From 1995 to 1997 , he joined the army of Taiwan. From 1997 to 2003, he joined the faculty of the $\mathrm{Wu}$ Feng Institute of Technology, Shu-Te University, and National Kaohsiung University of Applied Sciences, all in southern Taiwan. In 2004, he joined the faculty of the Department of Systems and Naval Mechatronic Engineering, National Cheng-Kung University, Tainan, Taiwan, where he is now an Associate Professor. His research interests include microwave imaging, antennas, microwave circuits, and application of wireless technologies in land, oceanic, and underwater environments.

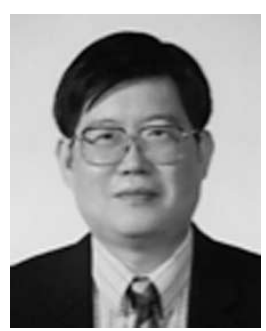

Tah-Hsiung Chu (M'87) received the B.S. degree from National Taiwan University, Taipei, Taiwan, R.O.C., in 1976, and the M.S. and Ph.D. degrees from the University of Pennsylvania, Philadelphia, in 1980 and 1983, respectively, all in electrical engineering.

From 1983 to 1986 , he was a Member of the Technical Staff with the Microwave Technology Center, RCA David Sarnoff Research Center, Princeton, NJ. Since 1986, he has been on the faculty of the Department of Electrical Engineering, National Taiwan University, where he is now a Professor of electrical engineering. His research interests include microwave-imaging systems and techniques, microwave circuits and subsystems, microwave measurements, and calibration techniques. 